\title{
High Order Sliding Mode Control for Suppression of Nonlinear Dynamics in Mechanical Systems with Friction
}

\author{
Rogelio Hernandez Suarez ${ }^{1}$, America Morales Diaz ${ }^{2}$, Norberto Flores \\ Guzman $^{3}$, Eliseo Hernandez Martinez ${ }^{4}$ and Hector Puebla ${ }^{4}$ \\ ${ }^{1}$ Instituto Mexicano del Petróleo, México D.F. \\ ${ }^{2}$ Centro de Investigación y Estudios Avanzados del IPN, Saltillo Coahuila \\ ${ }^{3}$ Centro de Investigación en Matemáticas, Guanajuato Guanajuato, Saltillo, Coahuila, \\ ${ }^{4}$ Universidad Autónoma Metropolitana, México D.F.
}

México

\section{Introduction}

Friction occurs in all mechanical systems, (e.g. bearings, transmissions, hydraulic and pneumatic cylinders, valves, brakes and wheels). Friction is the tangential reaction force between two surfaces in contact. There is a wide range of physical phenomena that cause friction, this includes elastic and plastic deformations, fluid mechanics and wave phenomena, and material sciences (Bowden \& Tabor, 1950; Armstrong-Hélouvry, 1994; Rabinowicz, 1995). In mechanical systems, friction can limit the performance in terms of increasing tracking errors and, under certain conditions, friction leads to oscillatory behavior, including simple periodic oscillations and chaos (Feeny \& Moon, 1994; Hikihara \& Moon, 1994; Ibrahim, 1994). In many practical situations it may be desirable that a given system originally undergoing complicated behavior should be forced to display regular motions (e.g., suppression of oscillatory dynamics). For instance, it could be desirable to induce regular dynamics in mechanical oscillators to avoid errors (as in the case of precise position mechanisms) lead by external vibrations and magnetic fields (Chatterjee, 2007; Fradkov \& Pogromsky, 1998; Southward et al., 1991).

To deal with systems with friction, it is necessary to have a good characterization of the structure of the friction model and then to design appropriate compensation techniques. As a friction phenomenon has not yet been completely understood, friction modeling is not an easy task. Indeed, uncertainty exists on most models that contain a friction component (Hinrichs et al., 1998; Feeny, 1998; Armstrong-Hélouvry et al., 1994; Olsson et al., 1998). Thus, for control design purposed for systems with friction it is necessary to consider the uncertainty of models that includes friction.

Different control approaches for friction compensation have been proposed. For instance, linear control system with type PI controllers (Puebla \& Alvarez-Ramirez, 2008), adaptive compensation (Canudas de Wit \& Lischinsky, 1997; Huang et al., 2000; Tomei, 2000), neural networks (Lin \& Wai, 2003), and others nonlinear model-based methods (Alvarez-Ramirez et 
al., 1995; Chatterjee, 2007; Xie, 2007; Zeng \& Sepehri, 2008). The use of linear techniques is very limiting due the highly nonlinearity of mechanical systems with friction, whereas nonlinear control design needs, in general, too much information about the process. The performance of a model-based adaptive control is limited by the accurateness of the model used to describe the various friction-related effects.

Sliding mode control is a robust control method that has long been applied in simple mechanical systems (Hangos et al., 2004). A drawback of this methodology, which usually limits its applicability to control mechanical systems, is the high-frequency switching of the control action which induces the so-called chattering phenomenon, i.e. undesired oscillations of the relevant signals causing vibrations and unacceptable mechanical wear (Hangos et al., 2004). A possibility to overcome this problem is to rely on high order sliding mode control (Laghrouche et al., 2007; Levant, 2005; Levant, 2001). In (Hernandez-Suarez et al., 2009) we have applied the integral high order sliding mode control (IHOSMC), to suppress stick-slip oscillation in an oil-drillstring. The resulting feedback control approach leads to a robust feedback control scheme that deals with uncertainties in the friction model and drillstrings parameters. The IHOSMC approach consider the integration of a fractional power of the absolute value of the tracking error, coupled with the sign function (Laghrouche et al., 2007; Aguilar-Lopez et al., 2010). This control structure provides simplification of the control law and good robustness properties. In this chapter we extend the application of IHOSMC approaches to control a general class of mechanical systems with friction. The proposed controller yield to a robust performance in presence of external disturbances and uncertainty on the parameters of systems with friction.

This chapter is organized as follows: In Section 2, for the sake of clarity in presentation, we briefly provide some issues on the friction phenomenology and modeling, and the class of mechanical systems with friction is also introduced. In Section 3 we present the IHOSMC approach and introduce a recursive cascade control scheme for the control of the class of mechanical systems considered in this chapter. Numerical benchmark examples are used to illustrate the control performance of the proposed control approach. Finally, in Section 6 we close this chapter with some concluding remarks.

\section{Mechanical systems with friction}

In this section we briefly discuss the friction phenomenology. Next we present some classical models of friction phenomena. Finally, we introduce the class of mechanical systems under consideration in this chapter.

\subsection{Friction phenomenology}

Friction involves two solid surfaces sliding against each other. The friction force is affected by many factors such as the properties of bulk and surface layer materials, the roughness of the surfaces in contact, the stress levels, the sliding speed, the temperature, the environment, the properties of the lubricants and the lubrication conditions. Lubrication has the main purpose of creating a fluid film between the two contacting surfaces, avoiding solid-to-solid contact (Bowden \& Tabor, 1950; Bowden \& Tabor, 1964; Armstrong-Hélouvry et al., 1994; Rabinowicz, 1995).

Friction is a torque, or a force, that depends on the relative velocity of the moving surfaces. Although there is disagreement on the character of the functionality of the friction forces with the velocity, experiments have confirmed that, for moderate and low velocities, the main 
components in the friction forces are mainly caused by the following phenomena (Bowden \& Tabor, 1950; Bowden \& Tabor, 1964; Armstrong-Hélouvry et al., 1994; Rabinowicz, 1995):

1. Coulomb and sticktion: Coulomb friction is due to sticking effects. At zero speed, the friction torque is equal and opposite of the applied torque, unless the latter one is larger than the stiction torque. In this case, the friction torque is equal to the stiction torque, $F_{s}$. The stiction torque is a torque at the moment of breakaway and is larger than the Coulomb torque. Kinetic friction $F_{r}$ is the resisting force which acts on a body after the force of static friction has been overcome.

2. Stribeck (downward bends): After the sticktion force has been overcame, the friction force decreases exponentially, reaching a minimum, and then increases proportionally with the velocity. These bends occur at velocities close to zero. The friction forces are due to a partial lubrication, where the velocity is adequate to entrain some fluid in the junction but not enough to fully separate the surfaces.

3. Viscous: Here the surfaces are fully separated by fluid film. In this regime the viscosity of the lubricant dominates and friction increases with velocity.

4. Asymmetries and position dependence: Imperfections and unbalances in the mechanism induce asymmetries and position dependence of the friction forces.

\subsection{Friction models}

Many friction models have been developed and reflect different aspects of the friction phenomena (Armstrong-Hélouvry et al., 1994; Hinrichs et al., 1998; Olsson et al., 1998). In general, existing friction models are usually classified as static and dynamic, where the fundamental difference between them is the frictional memory. Static models usually have a form of direct dependence between the friction force and relative velocity. Dynamic friction models where memory effect is described with a complimentary dynamics between the velocity and the friction force. Typical friction models are the Columb, Dahl, and the LuGre, friction models.

1. Columb model: Coulomb proposed the first model for the physical origin of friction, which explained some of the important properties of dry friction in a simple way. Coulomb sliding friction is given by,

$$
f=F_{N} f_{c} \operatorname{sign}(v) \quad v \neq 0
$$

where $f$ is a friction force, $F_{N}$ the normal load, $f_{c}$ the coefficient of Coulomb friction and $v$ is relative velocity. The indeterminate and discontinuous nature of the Coulomb model makes it extremely difficult to simulate the dynamics of the mechanical systems (Olsson et al, 1998).

2. Dahl model: Dahl developed a simple model, which can be considered as a generalization of Coulomb friction. The frictional hysteresis during pre-sliding is approximated by a generalized first order equation of the position depending only on the sign of the velocity. Dahl proposed the following equation,

$$
\frac{d f}{d t}=\sigma_{0}\left(1-\operatorname{sign}(v) \frac{F}{F_{s}}\right)^{\delta_{d}} v
$$

where $\sigma_{0}$ denotes the initial stiffness of the contact at velocity reversal and $\delta_{d}$ denotes a model parameter determining the shape of the hysteresis. $v$ is the relative moving speed. 
$F_{S}$ is the highest steady state friction. The Dahl model produces a smooth transition around zero velocity (Olsson et al, 1998; Dahl, 1976).

3. Stribeck friction: The Stribeck friction may be represented by,

$$
\left.f=F_{0} \operatorname{sign}(v)+F_{s} \exp \left(-\left(v / v_{S}\right)^{2}\right)-1\right) \operatorname{sign}(v)
$$

Where $F_{0}$ is the static friction, $F_{S}$ is the magnitude of the Stribeck effect, $v_{S}$ is the critical velocity of the Stribeck effect, and $v$ is the velocity. This model is empirical and in most cases has a good fit to data (Olsson et al, 1998).

4. LuGre model: The LuGre model describe major features of dynamic friction, including presliding displacement, varying break-away force and Stribeck effect. The LuGre model is given by,

$$
\begin{aligned}
f & =\sigma_{0} z+\sigma_{1} \dot{z}+\alpha_{2} v \\
\dot{z} & =v-\frac{|v|}{g(v)} z \\
g(v) & =\alpha_{0}+\alpha_{1} \exp \left(-\left(v / v_{s}\right)^{2}\right)
\end{aligned}
$$

where $z$ represents the unmeasurable internal friction state, $\sigma_{0}, \sigma_{1}, \alpha_{2}$ are parameters associated to the stiffness of the elastic bristle, damping coefficient in elastic range, and the viscous friction coefficient, respectively, $v$ is the relative velocity, and $v_{S}$ is the Stribeck velocity. The function $g(v)$ is positive and it describes the Stribeck effect. Direct use of the above LuGre model for friction compensation may have some implementation problems. Namely, as the internal friction state $z$ is unmeasurable, it is necessary to construct observers to estimate $z$ for dynamic friction compensation (Canudas de Wit et al., 1995; Olsson et al, 1998).

\subsubsection{Stick-slip}

This phenomenon consists on the sudden and successive change from "stick" state to "sliding" state, provoking the apparition of vibration and noise. Stick-slip friction is present in any elements involving relative motion, such as gears, pulleys, bearings, DC motors. Stick-slip friction is generally described as a composite of two different processes: the static process when an object is stationary (no sliding is involved) and likely to move under certain applied torque, and the dynamic process when sliding is involved (Fidlin, 2006; Denny, 2004).

The static process is characterized by the maximum static torque (or breakaway torque), under which static state remains and the magnitude of the static friction force is equal to that of the applied force. The slipping process is relatively complicated. Slipping torque is usually modeled as a linear combination of Coulomb torque, viscous torque, exponential torque used to represent Stribeck effect, and position dependent components (Fidlin, 2006; Denny, 2004). A mathematical formulation of the stick-slip friction (denoted by $\tau_{f m}$ ) with some commonly used friction components is (Fidlin, 2006; Denny, 2004),

$$
\begin{aligned}
\tau_{f m} & =\tau_{s t m} \operatorname{sign}(v)+k_{v i s} v-\tau_{c m}\left(1-\exp \left(-T_{0}|v|\right)\right) \operatorname{sign}(v) \\
& +\tau_{p m} \operatorname{sign}(v)+(1-\operatorname{sign}(v)) \tau_{i}
\end{aligned}
$$


where $k_{v i s}$ is the coefficient of viscous friction, $\tau_{s t m}$ represents the maximum static friction torque, the third term on the right side represents the Stribeck effect, where $T_{0}$ is a positive constant, $\tau_{i}$ is the input torque, so that the last term on the right side stands for the static friction force whose magnitude is equal to the applied force, and $\tau_{p m}$ is the position dependent friction torque, which can be modeled as,

$$
\tau_{p m}=\beta_{1} \sin \left(\beta_{2} q+\beta_{3}\right)
$$

where $\beta_{i}$ are constants.

\subsection{The class of mechanical systems with friction}

We consider a $n$ order generic simple model of a mechanical system with friction, which, possibly after a change of coordinates, can be described by,

$$
\begin{aligned}
\dot{x}_{i} & =f_{i}(x)+g_{i}(x) x_{i+1} \quad 1 \leq i \leq j-1 \\
\dot{x}_{j} & =f_{j}(x)+g_{j}(x) u \quad j \leq n \\
\dot{x}_{j+l} & =f_{j+l}(x) \quad j+l=n \quad \text { for } j<n
\end{aligned}
$$

where $x$ are the states of the system and $u$ is the control input. Note that the $j$ first equations in dynamical system (7) are in the so called chained form. Its not hard to see that several model of mechanical systems with friction can be described by (7). For instance, a basic model formulation, that covers various mechanical models reported in literature is described by,

$$
\ddot{x}=-F(\dot{x}, t)+u
$$

where $x$ is the position, $x$ is the speed, $F$ is a nonlinear function including friction components, and $u$ is an external input. This system can be described by (7) under a simple coordinates change.

\section{Integral high order sliding model control of mechanical systems with friction}

In this section, the IHOSMC is presented to control the class of mechanical systems described in the above section. By exploiting the chained form of model (7) we use a recursive cascade control configuration, where a virtual control input is introduced for the control design, and a single control input $u$, related to the electrical properties of the motor and consequently, the torque supplied by the motor is employed.

\subsection{Control problem}

The control objective is the regulation or tracking of an intermediate state $\left(x_{1} \leq x_{i} \leq j-1\right)$ of the class of mechanical systems with friction in the form (7) about a given reference, i.e. $x_{i} \rightarrow x_{i, r e f}$ under the following assumptions,

A1 States $x_{1}$ to $x_{j}$ are available for control design purposes.

A2 Nonlinear terms $f$ and $g$ and model parameters are uncertain, and can be available rough estimates of these terms. 


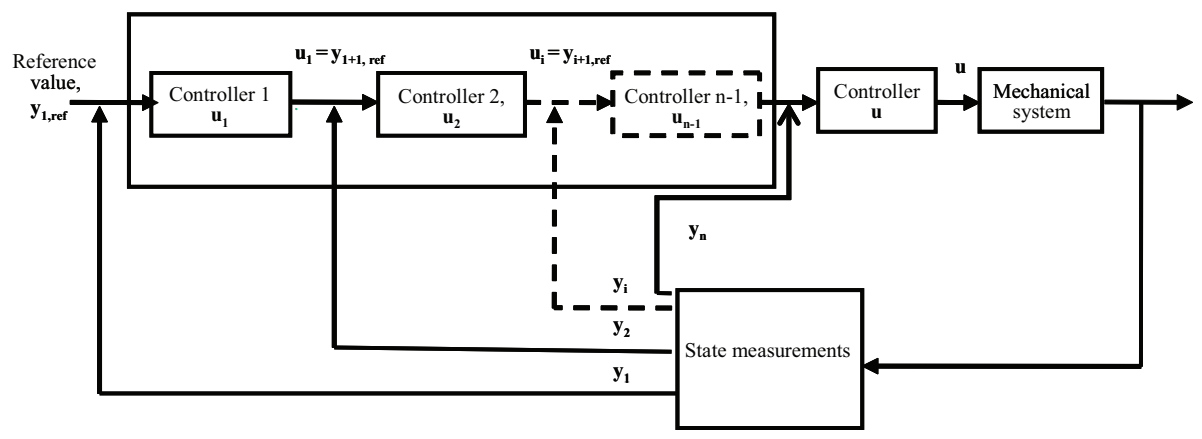

Fig. 1. Cascade control for mechanical systems with friction.

The following comments are in order:

- Dynamical and design problems in mechanical systems can be addressed using large finite element models, which give quantitative information and can help to give practical recommendations to circumvent mechanical problems. However, in practice, control and optimization techniques tend to be based on simple models. Indeed, for control systems design purposes, both low dimensional and less complex models can provide (to some degree) qualitative insight on the dominant complex phenomenon occurring in mechanical systems with friction.

- Assumption $A 1$ is a reasonable assumption in several applications. For instance, high precision optical encoders for position measurement. On the other hand, even in the absence of such measurements, a state estimator can be designed.

- A2 considers that nonlinear terms, including friction, can contain uncertain parameters, or in the worst case the whole terms are unknown. From a practical viewpoint, obtaining a model that can embody all such characteristics of the friction force is not an easy task. Having selected an appropriate model, the parameters of the model are needed to be experimentally identified to implement the model. Friction identification is another challenging part of the friction compensation process.

\subsection{A cascade control scheme}

We can exploit the structure of the model given by (7) to design a cascade procedure to control mechanical systems with friction. Cascade control is a common control configuration in process control, which can be thought of as partial state feedback. A typical cascade control structure has two feedback controllers with the output of the primary (master) controller changing the set point of the secondary (slave) controller (Alvarez-Ramirez et al., 2002; Krishnaswamy et al., 1990).

Figure 1 shows the recursive cascade control configuration for the class of mechanical systems given by (7). The cascade control configuration is based on the design of an intermediate virtual control function $u_{v i}=u_{i}$. The design is recursive because the computation of $u_{i+1}$ requires the computation of $u_{i}$. For instance, for the simple class of mechanical system (8), the master controller regulates the mechanical position $x_{1}$ to a desired reference $x_{1, r e f}$ with the virtual input $x_{2}=u_{v i}$, the slave controller regulates the velocity state variable $x_{2}$ to the reference $x_{2, \text { ref }}=u_{v i}$ with the real control input $u$. In other words, the master controller 
provides reference values $x_{2, r e f}$ to the slave controller, which is driven by the real control input $u$.

\subsection{Integral high order sliding mode control}

Sliding mode control techniques have long been recognized as a powerful robust control method (Hangos et al., 2004; Levant, 2001; Sira-Ramirez, 2002). Sliding mode control is a nonlinear controller due of the switching control action. Sliding-mode control schemes, have shown several advantages like allowing the presence of matched model uncertainties and convergence speed over others existing techniques as Lyapunov-based techniques, feedback linearization and extended linearization. However standard sliding-mode controllers have some drawbacks: the closed-loop trajectory of the designed solution is not robust even with respect to the matched disturbances on a time interval preceding the sliding motion, the classical sliding-mode controllers are robust in the case of matched disturbances only, the designed controller ensures the optimality only after the entrance point into the sliding mode. To try to avoid the above a relatively new kind of sliding-mode structures have been proposed as the named high-order sliding-mode technique, these techniques consider a fractional power of the absolute value of the tracking error coupled with the sign function, this structure provides several advantages as simplification of the control law, higher accuracy and chattering prevention (Hangos et al., 2004; Levant, 2001; Sira-Ramirez, 2002).

\subsubsection{Control design}

Sliding mode control designs consists of two phases. In the first phase the sliding surface is to be reached (reaching mode), while in the second the system is controlled to move along the sliding surface (sliding mode). In fact, these two phases can be designed independently from each other. Reaching the sliding surface can be realized by appropriate switching elements (Hangos et al., 2004).

Defining,

$$
\sigma(e)=e_{i}=y-y_{\text {ref }}
$$

as the sliding surface, we have that the continuous part of the sliding mode controller is given by,

$$
u_{e q, i}=-g_{i}(x)^{-1}\left(f_{i}(x)-\dot{y}_{i, r e f}\right)
$$

such that,

$$
\dot{\sigma}\left(e_{i}\right)=0
$$

where $\dot{y}_{i, r e f}$ is the time-derivative of the desired trajectory signal. In sliding mode, the controlled system satisfies the condition $d e_{i} / d t=0$, such that the tracking error will be driven to zero.

To force the system trajectory to converge to the sliding surface in the presence of both model uncertainties and disturbances, with chattering minimization and finite-time convergence, the sliding trajectory is proposed as (Levant, 2001; Aguilar-Lopez et al., 2010),

$$
u_{s l d, i}=-g_{i}(x)^{-1}\left[\delta_{1, i} e_{i}+\delta_{2, i} \int_{0}^{t}\left\{\operatorname{sign}\left(e_{i}\right)\left|e_{i}\right|^{1 / p}\right\} d \tau\right]
$$


where $\delta_{1, i}$ and $\delta_{2, i}$ are control design parameters. In essence, to achieve a zero tracking error all system trajectory must be forced to converge to $\sigma\left(e_{i}\right)$ in finite time and to remain on $\sigma\left(e_{i}\right)$ afterwards.

The complete IHOSMC is given by,

$$
u_{i}=u_{e q, i}+u_{s l d, i}=-g_{i}(x)^{-1}\left(f_{i}(x)-\dot{y}_{i, r e f}+\delta_{1, i} e_{i}+\delta_{2, i} \int_{0}^{t}\left\{\operatorname{sign}\left(e_{i}\right)\left|e_{i}\right|^{1 / p}\right\} d \tau\right)
$$

The synthesis of the above control law requires accurate knowledge of both $f_{i}(x)$ and $d y_{i, r e f} / d t$ to be realizable.

To enhance the robust performance of the above control laws, the uncertain terms $f_{i}(x)$ are lumped in single terms and compensated with a reduced-order observer (Alvarez-Ramirez, 1999).

Then, we define the modelling error function as follows,

$$
\eta_{i}=f_{i}(x)
$$

In order to get estimated values of the modelling error functions $\widetilde{\eta}$, we introduce the following reduced-order observer,

$$
\dot{\widetilde{\eta}}_{i}=\lambda_{i}\left(\eta_{i}-\widetilde{\eta}_{i}\right)
$$

where $\lambda_{i}$ are observer design parameters. From (7) and (15), and after of some direct algebraic manipulation we get,

$$
\dot{w}_{i}=-g_{i}(x) u_{i}-\widetilde{\eta}_{i} \quad \widetilde{\eta}_{i}=\lambda_{i}\left(w_{i}+y_{i}\right)
$$

The robust IHOSMC law is written as,

$$
u_{i}=-g_{i}(x)\left(\widetilde{\eta}_{i}-\dot{y}_{i, r e f}+\delta_{1, i} e_{i}+\delta_{2, i} \int_{0}^{t}\left\{\operatorname{sign}\left(e_{i}\right)\left|e_{i}\right|^{1 / p}\right\} d \tau\right)
$$

By exploiting the properties of the sliding part of the sliding-mode type controllers to compensates uncertain nonlinear terms, the knowledge of nonlinear terms $f_{i}(x)$ can be avoided. On the order hand, extensive simulation examples show that the derivative of the set point variable can be eliminated without affecting the closed loop system performance. Summarizing, the IHOSMC is composed by a proportional action, which has stabilizing effects on the control performance, and a high order sliding surface, which compensates the uncertain nonlinear terms to provide robustness to the closed-loop system. This behavior is exhibited because, once on the sliding surface, system trajectories remain on that surface, so the sliding condition is taken and make the surface and invariant set. This implies that some disturbances or dynamic uncertainties can be compensated while still keeping the surface an invariant set.

The following comments are in order:

- The IHOSMC approach has two control design parameters, namely, $\delta_{1}$ and $\delta_{2}$ which will be chosen as $\delta_{1}>\delta_{2}>0$. On the other hand, parameter $\lambda$, of the uncertainty observer should be chosen as $0<\lambda<\omega_{\mathcal{c}}$, where $\omega_{\mathcal{c}}$ is the open-loop dominant frequency of the systems oscillations. 
- Although a rigorous robustness analysis is beyond the scope of this study, numerical examples will show that the feedback controller is able of yielding robust control performance despite significant parameter departures from parameter nominal values.

- Stability must be preserved in the context of both structured uncertainties in the parameters as well as unstructured errors in modeling. A stability analysis for the proposed control configurations should parallel the steps reported in (Aguilar-Lopez et al., 2010) and (Aguilar-López \& Martínez-Guerra, 2008).

\section{Applications}

In this section, simulation results are presented for both position regulation and tracking of mechanical systems with friction (7) with the IHOSMC approach described above. The control performance is evaluated considering set point changes and typical disturbances of mechanical systems. We consider the following five examples: (i) Mechanical system with Coulomb friction, (ii) an inverted pendulum, (iii) an AC induction motor, and (vi) a levitation magnetic system.

\subsection{Mechanical system with Coulomb friction}

We consider a mechanical system described in (Alvarez-Ramirez et al., 1995) with a Coulomb friction law. The dimensionless equation of motion is,

$$
\begin{aligned}
& \frac{d x_{1}}{d t}=x_{2} \\
& \frac{d x_{2}}{d t}=\frac{1}{m}\left\{-F\left(x_{1}, x_{2}\right)-\alpha x_{1}+\tau_{l}+u\right\}
\end{aligned}
$$

where $m$ is the mass of the system, $\tau_{l}$ is an unknown external force, which way be due to loads and/or noise acting in the mechanism, $u$ is a manipulated forced used to control the system and the term $F\left(x_{1}, x_{2}\right)$ includes all friction effects and is determined by the following expression,

$$
F\left(x_{1}, x_{2}\right)=\phi f\left(x_{1}\right)
$$

where $\phi$ is the coefficient of friction and $f\left(x_{1}\right)$ is the normal load which vary with displacement,

$$
\begin{aligned}
f\left(x_{1}\right) & =-\mu_{k} \quad x_{1}<0 \\
-\mu_{s} & \leq f\left(x_{1}\right) \leq \mu_{s} x_{1}=0 \\
f\left(x_{1}\right) & =\mu_{k} \quad x_{1}>0
\end{aligned}
$$

Control objetive is the position tracking to the periodic reference,

$$
y_{\text {ref }}=x_{1, \text { ref }}=0.3 \sin (0.5 t)
$$

The parameters of the controller are set as $\delta_{1, i}=[25,10]$, and $\delta_{2, i}=[2.3,1]$. Model simulation parameters are taken from (Alvarez-Ramirez et al., 1995). The control law is turned on the $t=50$ time units and $\tau_{l}=A \sin (1.25 t)$. At $t=75$ the amplitude $A$ of the external force $\tau_{l}$ is 

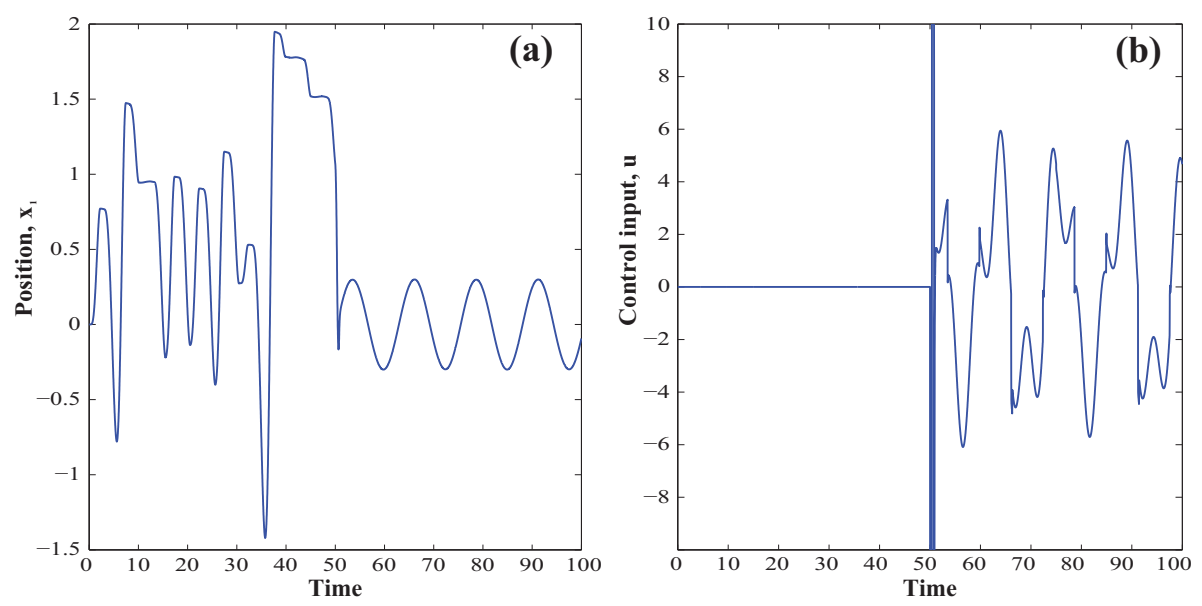

Fig. 2. (a) Cascade control for mechanical system, (18) and (b) control input.

changed in $20 \%$. Figure 2 shows the position trajectory before and after the control activation. In Figure 2 the control input is also displayed. It can be seen that the proposed cascade control scheme is able to track the desired reference and rejects the applied perturbation. After that the control input reach the saturation levels $(-10<u<10)$ the control inputs displays a complex oscillatory behavior.

\subsection{Inverted pendulum}

The inverted pendulum has been used as a classical control example for nearly half a century because of its nonlinear, unstable, and nonminimum-phase characteristics. In this case we consider a single inverted pendulum.

The equation of motion for a simple inverted pendulum with Coulomb friction and external perturbation is (Poznyak et al., 2006),

$$
\begin{aligned}
& \frac{d x_{1}}{d t}=x_{2} \\
& \frac{d x_{2}}{d t}=-g \sin \left(x_{1}\right) / l-v_{s} x_{2} / J-p_{s} \operatorname{sign}\left(x_{2}\right) / J+\tau_{d}+u / J
\end{aligned}
$$

where $g$ is the gravitational acceleration, $l$ is the distance between the rotational axis and center of gravity of the pendulum, $J=m l^{2}$ is the inertial moment, where $m$ is the mass of the system, $\tau_{d}=0.5 \sin (2 t)+0.5 \cos (5 t)$ is an external disturbance, which may be due to loads and/or noise acting in the mechanism, $u$ is a manipulated forced used to control the system. Let $y_{\text {ref }}=x_{1, r e f}=\sin (t)$ be the desired orbit of the pendulum position. Figure 3 shows the control performance using the control parameters $\delta_{1, i}=[12,7]$, and $\delta_{2, i}=[1,0.5]$. In this case the IHOSMC controller is activated at $t=15$ and from 0 to 15 time units the pendulum is driven by the twisting controller introduced by Poznyak et al. (2006). It can be seen from Figure 3 that the IHOSMC controller is able to follow the periodic orbit with a better closed loop behavior that the twisting controller. 

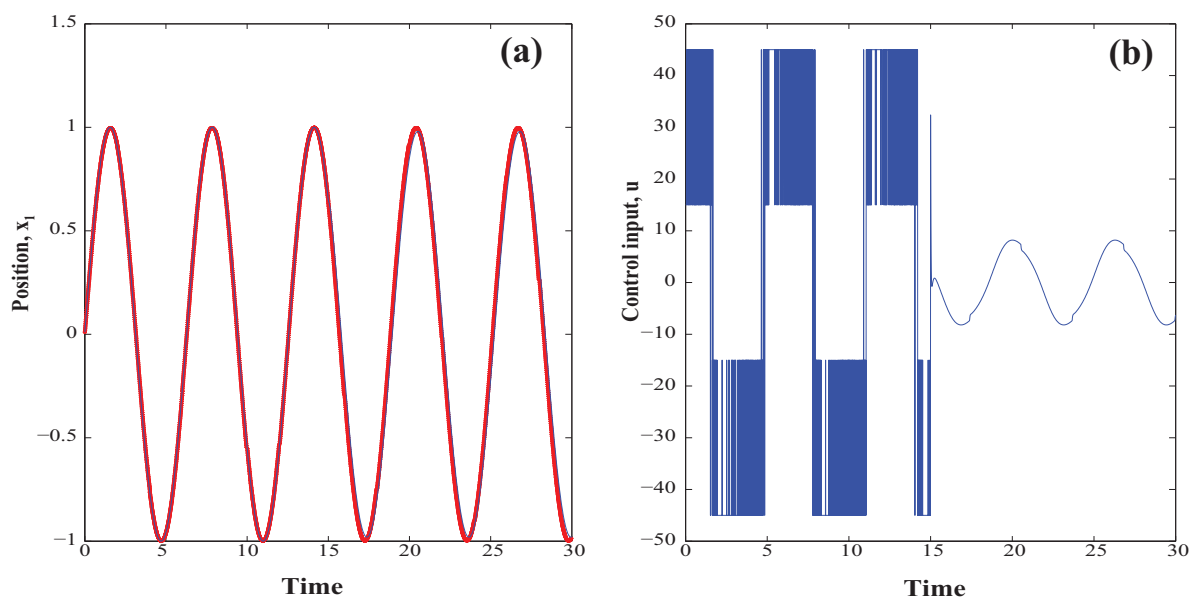

Fig. 3. (a) Control performance for inverted pendulum system and (b) control input.

\subsection{Induction AC motors}

Induction motors have found considerable applications in industry due to their reliability, ruggedness and relatively low cost. Their mechanical reliability is due to the fact that there is no mechanical commutation as in most DC motors. Furthermore, induction motors can also be used in volatile environments because no sparks are produced. An induction motor is composed of three stator windings and three rotor windings.

A simple mathematical model of an induction motor, under field-oriented control with a constant rotor flux amplitude, which was presented in (Tan et al., 2003), is the following,

$$
\begin{aligned}
& \frac{d x_{1}}{d t}=x_{2} \\
& \frac{d x_{2}}{d t}=\frac{K_{T}}{J} x_{3}-\frac{F}{J}-\frac{\tau_{l}}{J} \\
& \frac{d x_{3}}{d t}=a_{1} x_{2}+a_{2} x_{3}+b u
\end{aligned}
$$

where $x_{1}$ is the rotor angle, $x_{2}$ is the rotor angular velocity, $x_{3}$ is the component of stator current, $u$ is the component of stator voltage, $J$ is the rotor inertia, $\tau_{l}$ is the load torque, and $F$ is the friction force.

Friction force is modeled by the LuGre friction model with friction force variations,

$$
\begin{aligned}
\frac{d z}{d t} & =x_{2}-\frac{\left|x_{2}\right|}{g\left(x_{2}\right)} z \\
F & =\sigma_{0} z+\sigma_{1} \frac{d z}{d t}+\sigma_{2} x_{2}
\end{aligned}
$$



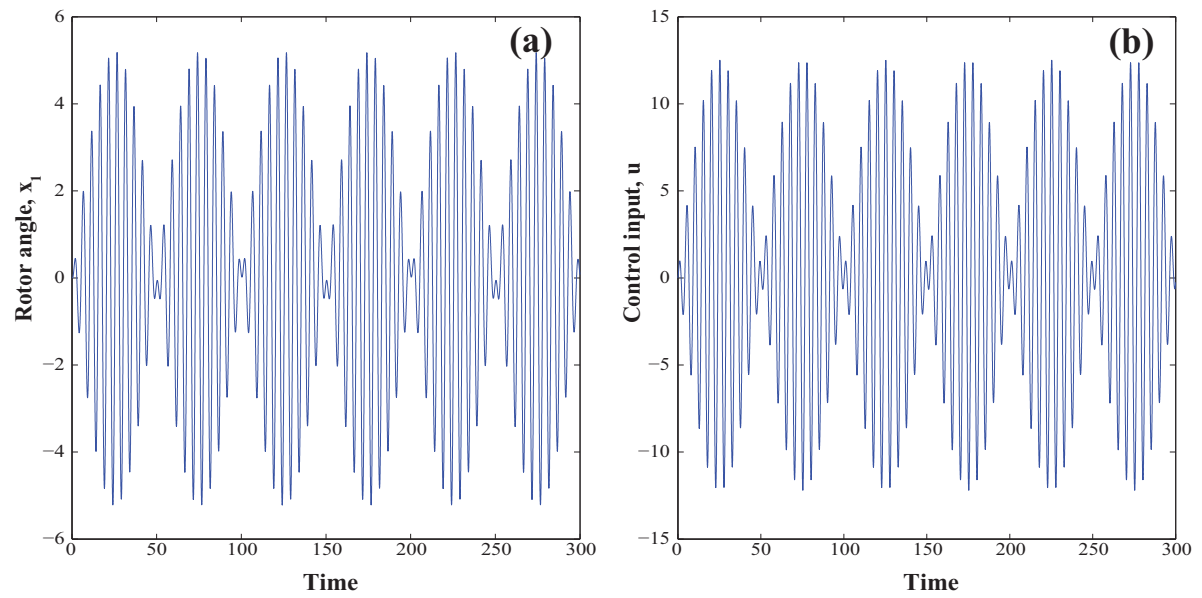

Fig. 4. (a) Cascade control for induction AC motors system and (b) control input.

where $z$ is the friction state that physically stands for the average deflection of the bristles between two contact surfaces. The nonlinear function is used to describe different friction effects and can be parameterized to characterize the Stribeck effect,

$$
g\left(x_{2}\right)=F_{c}+\left(F_{s}-F_{c}\right) \exp \left(-\frac{x_{2}}{v_{s}}\right)^{2}
$$

where $F_{c}$ is the Coulomb friction value, $F_{S}$ is the stiction force value, and $v_{S}$ is the Stribeck velocity.

The control objective is to asymptotically track a given bounded reference signal $y_{\text {ref }}=x_{1, \text { ref }}$ given by,

$$
y_{\text {ref }}=5.6 \sin (0.4 \pi t) \sin (0.02 \pi t)
$$

A load disturbance $\tau_{l}=0.8 \mathrm{~N} \cdot \mathrm{m}$ is injected into the induction motor simulation model. The position of the rotor angle and the corresponding control input are shown in Figure 4. It can be seen that the controller is able to track the desired reference (24) using a periodic input of the control input. The external disturbance is also rejected without an appreciable degradation of the closed-loop system.

\subsection{Levitation system}

Magnetic levitation systems have been receiving considerable interest due to their great practical importance in many engineering fields (Hikihara \& Moon, 1994). For instance, high-speed trains, magnetic bearings, coil gun and high-precision platforms. We consider the control of the vertical motion in a class of magnetic levitation given by a single degree of freedom (specifically, a magnet supported by a superconducting system). In particular, we consider a magnet supported by superconducting system which can be represented by a second-order differential equation with a nonlinear term which involves hysteresis and periodic external excitation force. Without loss of generality, one can consider that the model of the levitation system is modelled by the following equation (Femat, 1998), 


$$
\begin{aligned}
& \frac{d x_{1}}{d t}=x_{2} \\
& \frac{d x_{2}}{d t}=-\delta x_{2}-x_{1}+x_{3}+\tau_{l}+u \\
& \frac{d x_{3}}{d t}=-\gamma\left(x_{3}-F\right)
\end{aligned}
$$

$x_{1}$ is defined as a displacement from the surface of a high Tc superconductor (HTSC) surface, $x_{2}$ is the velocity, $x_{3}$ is a dynamical force between the HTSC and the magnet, which includes hysteresis effects, $\delta$ represents a mechanical damping coefficient, $\gamma$ is a relaxation coefficient, $\tau_{l}$ is an external excitation force, and $u$ is the control force.

The nonlinear function $F$ is given by (Femat, 1998),

$$
\begin{aligned}
F & =F_{x_{1}} \exp \left(-x_{1}\right)\left(1-F_{x_{2}}\right) \\
F_{x_{1}} & =F_{0} \exp \left(-x_{1}\right) \\
F_{x_{1}} & =\left\{\begin{array}{cc}
-\mu_{1}-x_{2} & \epsilon \leq x_{2} \\
\frac{-x_{2}\left(\mu_{1}-\mu_{2}\right)}{2 \epsilon} & -\epsilon \leq x_{2}<\epsilon \\
\mu_{2} & x_{2}<-\epsilon
\end{array}\right.
\end{aligned}
$$

where the exponential term $F_{x_{1}}$ shows the force-displacement relation without hysteresis, $F_{0}$ denotes the maximum force between the HTSC and the magnet, $\mu_{1}$ and $\mu_{2}$ are constants. The control problem is the regulation to the origin of the vertical motion, i.e. $y_{\text {ref }}=x_{1, \text { ref }}=0.0$. In the Figure 6 the controlled position and the corresponding control input are presented (control action is turn on a $t=100.0$ time units). It can be seen from Figure 5 that the controller can regulate the vertical position of the levitation system via a simple periodic manipulation of the control force. The control input reaches saturation levels in the first 20 time units, which can be related to high values of the controller parameters.

\section{Conclusions}

In mechanical systems, the control performance is greatly affected by the presence of several significant nonlinearities such as static and dynamic friction, backlash and actuator saturation. Hence, the productivity of industrial systems based on mechanical systems depend upon how control approaches are able to compensate these adverse effects. Indeed, fiction in mechanical systems can lead to premature degradation of highly expensive mechanical and electronic components. On the other hand, due to uncertainties and variations in environmental factors a mathematical model of the friction phenomena present significant uncertainties.

In this chapter, by means of an IHOSMC approach and a cascade control configuration we have derived a robust control approach for both regulation and tracking position in mechanical systems. The underlying idea behind the control approach is to force the error dynamics to a sliding surface that compensates uncertain parameters and unknown term. The sliding mode control law is enhanced with an uncertainties observer. We have show via numerical simulations how the motion can be regulate and tracking to a desired reference in presence of uncertainties in the control design and changes in model parameters. Although 

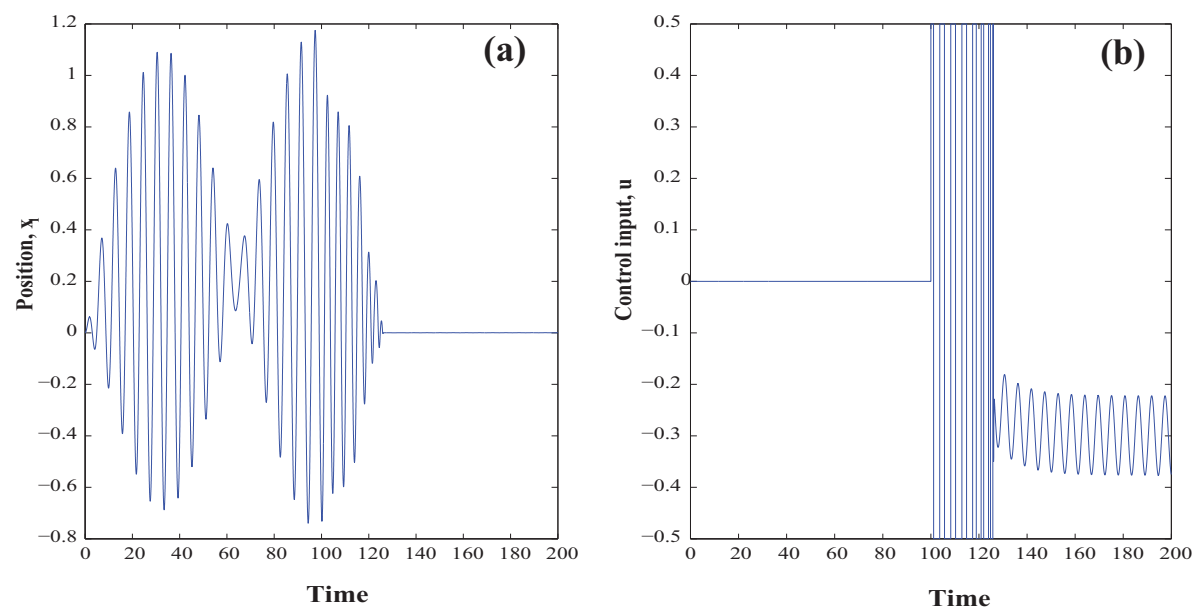

Fig. 5. Levitation system: (a) motion vertical control and (b) control input.

the control design is restricted to certain class of mechanical systems with friction, the concepts presented in our work should find general applicability in the control of friction in other systems.

\section{References}

Aguilar-Lopez, R.; Martinez-Guerra, R.; Puebla, H. \& Hernadez-Suarez, R. (2010). High order sliding-mode dynamic control for chaotic intracellular calcium oscillations. Nonlinear Analysis B: Real World Applications, 11, 217-231.

Aguilar-Lopez, R. \& Martinez-Guerra, R. (2008). Control of chaotic oscillators via a class of model free active controller: suppression and synchronization. Chaos Solitons Fractals, 38, No. 2, 531-540.

Alvarez-Ramirez, J. (1999). Adaptive control of feedback linearizable systems: a modelling error compensation approach. Int. J. Robust Nonlinear Control, 9, 361.

Alvarez-Ramirez, J.; Alvarez, J. \& Morales, A. (2002). An adaptive cascade control for a class of chemical reactors, International Journal of Adaptive Signal and Processing, Vol. 16, 681-701.

Alvarez-Ramirez, J.; Garrido, R. \& Femat, R. (1995). Control of systems with friction. Physics Review E, 51, No 6, 6235-6238.

Armstrong-Hélouvry, B.; Dupont, P. \& Canudas de Wit, C. (1994). A survey of models, analysis tools and compensations methods for the control of machines with friction. Automatica, 30, No. 7, 1083-1138.

Bowden, F.P. \& Tabor, D. (1950). The Friction and Lubrication of Solids. Oxford Univ. Press, Oxford.

Bowden, F.P. \& Tabor, D. (1964). The Friction and Lubrication of Solids: Part II. Oxford Univ. Press, Oxford.

Canudas de Wit, C. \& Lischinsky, P. (1997). Adaptive friction compensation with partially known dynamic friction model. Int. J. Adaptive Control Signal Processing, Vol. 11, 65-80. 
Canudas de Wit, C.; Olsson, H.; Astrom, K.J. \& Lischinsky, P. (1995). A new model for control of systems with friction. IEEE Tran. Automatic Control, 40, No. 3, 419-425.

Chatterjee, S. (2007). Non-linear control of friction-induced self-excited vibration. Int. J. Nonlinear Mech., 42, No. 3, 459-469.

Dahl, P.R. (1976). Solid friction damping of mechanical vibrations. AIAA J., 14, No. 12, 1675-1682.

Denny, M. (2004). Stick-slip motion: an important example of self-excited oscillation. European J. Physics, 25, 311-322.

Feeny, B. \& Moon, F.C. (1994). Chaos in a forced dry-friction oscillator: experimental and numerical modelling. J. Sound Vibration, 170, No. 3, 303-323.

Feeny, B. (1998). A nonsmooth coulomb friction oscillator. Physica D, 59, 25-38.

Femat, R. (1998). A control scheme for the motion of a magnet supported by type-II superconductor. Physica D, 111, 347-355.

Fidlin, A. (2006). Nonlinear Oscillations in Mechanical Engineering. Springer, Berlin, Heidelberg, 2006.

Fradkov, A.L. \& Pogromsky, A.Y. (1998). Introduction to Control of Oscillations and Chaos. World Scientific Publishing.

Hangos, K.M.; Bokor, J. \& Szederkényi, G. (2004). Analysis and Control of Nonlinear Process Systems. Springer-Verlag London.

Hernandez-Suarez, R.; Puebla, H.; Aguilar-Lopez, R. \& Hernandez-Martinez, E. (2009). An integral high-order sliding mode control approach for controlling stick-slip oscillations in oil drillstrings. Petroleum Science Technology, 27, 788-800.

Hikihara, T. \& Moon, F.C. (1994), Chaotic levitated motion of a magnet supported by superconductor. Phys. Lett. A, 191, 279.

Hinrichs, N.; Oestreich, M. \& Popp, K. (1998). On the modelling of friction oscillators. Journal Sound Vibration, 216, No. 3, 435-459.

Huang, S.N.; Tan, K.K. \& Lee, T.H. (2000). Adaptive friction compensation using neural network approximations. IEEE Trans. Syst., Man and Cyber.-C, 30, No. 4, 551-557.

Ibrahim, R.A. (1994). Friction-induced vibration, chatter, squeal and chaos: part I: mechanics of contact and friction. Applied Mechanics Reviews, 47, No. 7, 209-226.

Krishnaswamy, P.R.; Rangaiah, G.P.; Jha, R.K. \& Deshpande, P.B. (1990). When to use cascade control. Industrial Engineering Chemistry Research, 29, 2163-2166.

Laghrouche, S.; Plestan, F. \& Glumineau, A. (2007). Higher order sliding mode control based on integral sliding mode. Automatica, 45, 531-537.

Levant, A. (2005). Homogeneity approach to high-order sliding mode design. Automatica, 41, 823- 830.

Levant, A. (2001). Universal SISO sliding-mode controllers with finite-time convergence. IEEE Trans. Automat. Contr., 46, 1447-1451.

Lin, F.J. \& Wai, R.J. (2003). Robust recurrent fuzzy neural network control for linear synchronous motor drive system. Neurocomputing, 50, 365-390.

Olsson, H.; Åström, K. J.; Canudas de Wit, C.; Gäfvert, M. \& Lischinsky, P. (1998). Friction models and friction compensation. Eur. J. Control, 4, No. 3, 176-195.

Poznyak, A.; Shtessel, Y.; Fridman, L.; Davila, J. \& Escobar, J. (2006). Identification of parameters in dynamic systems via sliding-mode techniques. In: Advances in Variable Structure and Sliding Mode Control, LNCIS, Vol. 334, 313-347.

Puebla, H.; Alvarez-Ramirez, J. \& Cervantes, I. (2003). A simple tracking control for chuas circuit. IEEE Trans. Circ. Sys. , 50, 280. 
Puebla, H. \& Alvarez-Ramirez, J. (2008). Suppression of stick-slip in oil drillstrings: a control approach based on modeling error compensation. Journal Sound Vibration, 310, 881-901,

Rabinowicz, E. (1995). Friction and Wear of Materials. New York, Wiley, Second edition.

Sira-Ramirez, H. (2002). Dynamic second order sliding-mode control of the Hovercraft vessel. IEEE Trans. Control Syst. Tech., 10, 860-865.

Southward, S.C.; Radcliffe, C.J. \& MacCluer, C.R. (1991). Robust nonlinear stick-slip friction compensation. Journal Dynamic Systems, Measurement, and Control, Vol. 113, 639-645.

Tan, Y.; Chang, J. \& Hualin, T. (2003). Adaptive backstepping control and friction compensation for AC servo with inertia and load uncertainties. IEEE Tran. Industrial Electronics, 50, No. 5, 944-952.

Tomei, P. (2000). Robust adaptive friction compensation for tracking control of robot manipulators. IEEE Trans. Automat. Contr., 45, No. 11, 2164-2169.

Xie, W.F. (2007). Sliding-mode-observer-based adaptive control for servo actuator with friction. IEEE Trans. Ind. Elec., 54, No. 3.

Zeng, H. \& Sepehri, N. (2008). Tracking control of hydraulic actuators using a LuGre friction model compensation. Journal Dynamic Systems Measurement Control, 30. 


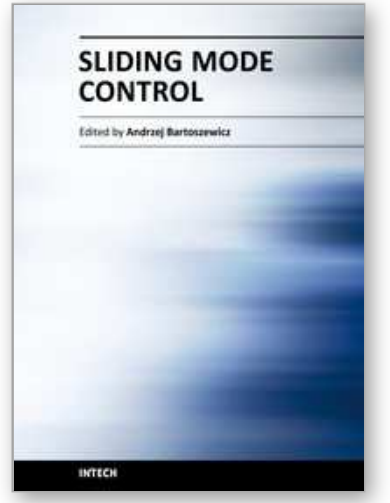

\author{
Sliding Mode Control \\ Edited by Prof. Andrzej Bartoszewicz
}

ISBN 978-953-307-162-6

Hard cover, 544 pages

Publisher InTech

Published online 11, April, 2011

Published in print edition April, 2011

The main objective of this monograph is to present a broad range of well worked out, recent application studies as well as theoretical contributions in the field of sliding mode control system analysis and design. The contributions presented here include new theoretical developments as well as successful applications of variable structure controllers primarily in the field of power electronics, electric drives and motion steering systems. They enrich the current state of the art, and motivate and encourage new ideas and solutions in the sliding mode control area.

\title{
How to reference
}

In order to correctly reference this scholarly work, feel free to copy and paste the following:

Rogelio Hernandez Suarez, America Morales Diaz, Norberto Flores Guzman, Eliseo Hernandez Martinez and Hector Puebla (2011). High Order Sliding Mode Control for Suppression of Nonlinear Dynamics in Mechanical Systems with Friction, Sliding Mode Control, Prof. Andrzej Bartoszewicz (Ed.), ISBN: 978-953-307-162-6, InTech, Available from: http://www.intechopen.com/books/sliding-mode-control/high-order-sliding-modecontrol-for-suppression-of-nonlinear-dynamics-in-mechanical-systems-with-fri

\section{INTECH}

open science | open minds

\section{InTech Europe}

University Campus STeP Ri Slavka Krautzeka 83/A 51000 Rijeka, Croatia Phone: +385 (51) 770447

Fax: +385 (51) 686166 www.intechopen.com

\section{InTech China}

Unit 405, Office Block, Hotel Equatorial Shanghai No.65, Yan An Road (West), Shanghai, 200040, China 中国上海市延安西路65号上海国际贵都大饭店办公楼405单元 Phone: +86-21-62489820

Fax: +86-21-62489821 
(C) 2011 The Author(s). Licensee IntechOpen. This chapter is distributed under the terms of the Creative Commons Attribution-NonCommercialShareAlike-3.0 License, which permits use, distribution and reproduction for non-commercial purposes, provided the original is properly cited and derivative works building on this content are distributed under the same license. 